\title{
An approved frequency tracking method in connected element interferometry
}

\author{
Qiang Zhao*, Kang Gao, and Xiaomin Hou \\ Department of Optical and Electronic Equipment, Aerospace Engineering University, Beijing, 101416, PR China
}

\begin{abstract}
Carrier tracking loop is a basic method of frequency tracking in CEI (connected element interferometry), but the tracking error is very large or even worse at high dynamic. To solve this problem, a frequency tracking method assisted by open loop frequency estimation is proposed in this paper. First, an open-loop frequency estimation algorithm is used to estimate the frequency of signals at different moments, then, the least squares fitting method is used to estimate the corresponding dynamic parameters of different orders. Next, a local signal is generated to compensate the received signal. After eliminating most of the dynamic characteristics, the signal is fed into PLL (Frequency locked loop) and high-precision frequency tracking results can be obtained. Simulation results show that, in the condition of low SNR, the new method has higher accuracy than the method assisted by FLL (Frequency locked loop), and the processing time is shorter. When there is a two order Doppler frequency change, the open-loop assisted method can overcome the steady phase error of the PLL, and is of great significance in the applications of connected elements interferometry.
\end{abstract}

\section{Introduction}

CEI is a method of target azimuth measurement, which has the advantages of high accuracy, short baseline and flexible station arrangement [1]. However, it has a very strict requirement for the measurement accuracy of signal frequency and phase [2]. Frequency tracking loop is a common method, but its accuracy is difficult to meet the requirements under high dynamic condition [3-5]. How to balance the contradiction between dynamic stress and tracking accuracy is a difficult and hot issue. At present, the carrier tracking method under high dynamic condition including the method based on kalman filter, the method assisted by FLL, and the method assisted by maximum likelihood estimation. Among them, kalman filter is used to estimate carrier phase difference, Doppler frequency and its change rate, and can adaptively adjust the loop parameters, but the uncertainty of the environment factors on the stability of the system greatly, and the computation is high, not easy to be implemented [6,7]. FLL has good resistance to dynamic stress, is responsible for eliminating most of the dynamic characteristics, but its tracking accuracy is low. The principle of this method is simple, but because of the large bandwidth of FLL loop, more noise is introduced into the tracking loop [8,9]. Maximum likelihood estimation is a kind of asymptotically unbiased estimation. The variance of parameter estimation can be close to the Cramer-rao lower bound (CRLB), but the storage and computation are relatively large, and the real-time application is greatly restricted $[10,11]$. This paper introduces the least square fitting method $[12,13]$, to predict continuous change of signal frequency by estimating signal frequency of partial period. And then the dynamic characteristic parameters of Doppler can be calculated, on this basis, generating a local signal to compensate Doppler frequency and eliminate most of the dynamic characteristics of the received signal. Finally accurate synchronization is finished by PLL.

\section{Traditional frequency tracking loop in CEI}

PLL is the common frequency tracking loop in CEI. The tracking error of PLL loop caused by thermal noise is [14]:

$$
\sigma_{T}=\frac{1}{2 \sqrt{2} \pi \tau} \sqrt{\frac{N_{0}}{P_{s}} B_{L}}
$$

In formula (1), $\tau$ is integration time, $N_{0}$ is noise power spectral density, $P_{s}$ is signal power and $B_{L}$ is bandwidth of loop filter.

It can be seen from the formula (1) that the loop bandwidth determines the tracking accuracy when integration time is fixed. In high dynamic conditions, in order to ensure that the loop does not mismatch, a larger loop bandwidth is generally set, which to some extent sacrifice the tracking accuracy. On the other hand, when there is a two order Doppler frequency change rate, the three order PLL is usually used. At this point, there will be a steady phase error. Assuming the two order Doppler

\footnotetext{
* Corresponding author: zqaniu@163.com
} 
frequency jerk is $R_{2}$, acceleration is $R_{1}$ and initial value is $f_{d 0}$, the instantaneous frequency of the signal can be expressed as a formula (2):

$$
f_{d}=1 / 3 R_{1} t^{2}+1 / 2 R_{2} t+f_{d 0}
$$

After the derivation, when the frequency has a parabolic variation, the steady phase error of the three order PLL is:

$$
\begin{aligned}
\theta_{e}(\infty) & =\lim _{s \rightarrow 0} s \bullet \theta_{e}(s)=\lim _{s \rightarrow 0} s \cdot H_{e}(s) \cdot \theta_{1}(s) \\
& =\lim _{s \rightarrow 0} s \cdot \frac{s^{3}}{s^{3}+s^{2} K a+s K b+K c} \cdot \frac{2 R_{2}}{s^{4}} \\
& =\frac{2 R_{2}}{K c}
\end{aligned}
$$

In above formula, $H_{e}(s)$ is error transfer function, $\theta_{1}(s)$ is Laplace transform of input signal, $K$ is gain of loop, $a, b, c$ represent the coefficients of the three order loop filter respectively. $c=m \bullet \xi$,generally, $m=5$, $\xi=0.707$ (damping coefficient).From formula (4), when the filter coefficient and loop gain are fixed, the larger the jerk, the larger the steady-state phase error.In CEI, it's an important error term and must be elimanated.

In order to improve the frequency tracking precision under high dynamic conditions, the PLL tracking method assisted by FLL is more widely used in space TT \& C, and its principle is shown in figure 1 .

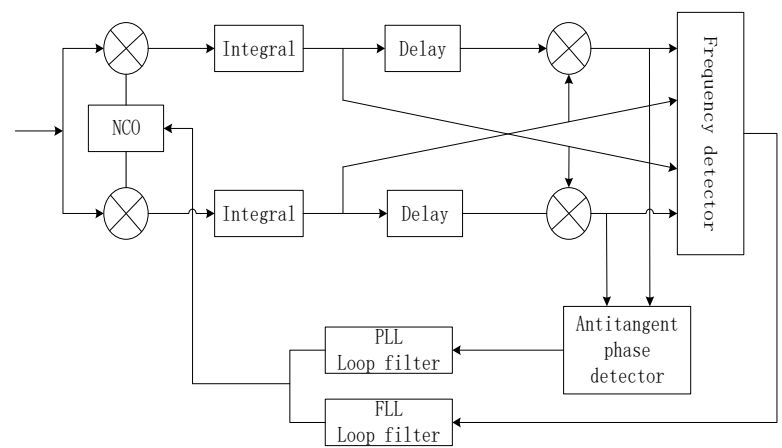

Figure.1. Principle of FLL-PLL frequency tracking loop

FLL is used to eliminate most of the dynamic structure the PLL determines the final tracking accuracy in Figure 1. The structure of this method is relatively simple, but because the FLL loop bandwidth is larger, PLL will also introduce more noise power. When SNR is low the tracking accuracy is low or even lock will be lost.

\section{High dynamic frequency tracking algorithm assisted by open loop frequency estimation}

In this paper, a high dynamic frequency tracking algorithm based on Rife open loop frequency estimation algorithm and least squares fitting is designed.

\subsection{Rife algorithm}

Rife algorithm is a classical open-loop frequency estimation algorithm for sinusoidal signals $[15,16]$, which effectively overcomes the fence effect of the

traditional DFT and improves the estimation accuracy greatly. Its expressions is as follows:

$$
\hat{f_{c}}=\Delta f \cdot\left(k_{0}-1+\alpha \cdot \frac{\left|G_{k_{0}+\alpha}\right|}{\left|G_{k_{0}+\alpha}\right|+\left|G_{k_{0}}\right|}\right)
$$

In formula (4), $\Delta f$ is frequency resolution of FFT, $k_{0}$ is the corresponding position of maximum spectrum, $G_{k_{0}}$ is the maximum value, $G_{k_{0}+\alpha}$ is sub large value of spectrum, $\alpha=1$ or -1 .

\subsection{Least squares fitting}

The idea of least square fitting is expressed as formula (5):

$$
\min _{\varphi} \sum_{i=1}^{m} \delta_{i}^{2}=\sum_{i=1}^{m}\left(\varphi\left(x_{i}\right)-y\right)^{2}
$$

Assuming that the fitting polynomial is

$$
y=a_{0}+a_{1} x+\cdots+a_{k} x^{k}
$$

Then

$$
\left[\begin{array}{cccc}
1 & x_{1} & \cdots & x_{1}^{k} \\
1 & x_{2} & \cdots & x_{2}^{k} \\
1 & \vdots & \ddots & x_{3}^{k} \\
1 & x_{n} & \cdots & x_{4}^{k}
\end{array}\right]\left[\begin{array}{c}
a_{0} \\
a_{1} \\
\vdots \\
a_{k}
\end{array}\right]=\left[\begin{array}{c}
y_{1} \\
y_{2} \\
\vdots \\
y_{n}
\end{array}\right]
$$

It can be written as $X * A=Y$, $A=\left(X^{\prime} * X\right)-1 * X^{\prime} * \mathrm{Y}$, thus, the coefficient matrix $A$ can be obtained. Furthermore, the fitting curve can be obtained.

The principle of high dynamic frequency tracking algorithm based on Rife algorithm and least square fitting designed in this paper as shown in figure 2 .

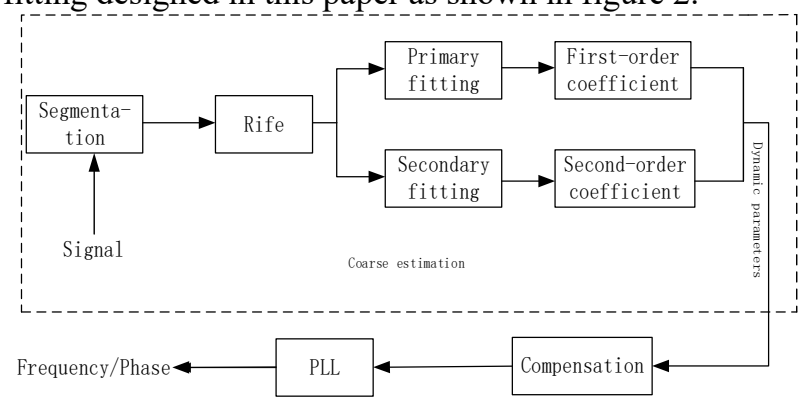

Figure.2. Principle of improved frequency tracking loop

The basic idea is to estimate the instantaneous frequency of different sampling time by using the high precision open loop frequency estimation algorithm, then, the curve of Doppler frequency with time is obtained by fitting the frequency at different time points by least square fitting method. At this time, the Doppler frequency dynamic parameters of different orders can be estimated. These coefficients are used to construct the local signal to compensate the received signal, eliminate most of the dynamic. At last, PLL is used to get a high accuracy frequency tracking result.

\section{Simulation experiment}




\subsection{High dynamic model and simulation parameters}

The high dynamic model of JPL is adopted in this paper. JPL proposed that the high dynamics is a relative concept, and considered that when the speed and acceleration value of the target is relatively large in reality, it is the high dynamic situation ${ }^{[4]}$, and the high dynamic model given by it is shown in figure 3 .
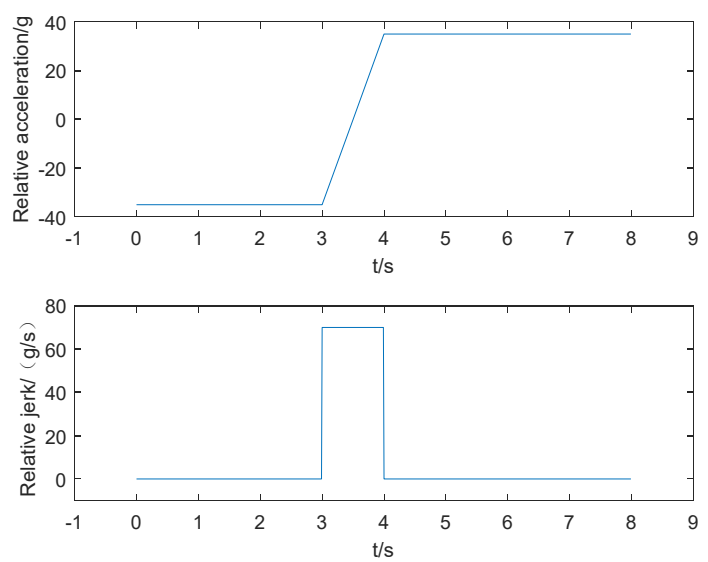

Figure.3. High dynamic acceleration model of JPL

In Figure 3, the acceleration corresponds to the first order change rate of the Doppler frequency, and the jerk rate corresponds to the two order change rate of the Doppler frequency. In practice, although there may be three or even higher order information, but the value is generally very small, therefore, only two order change rate is considered.

According to the JPL model, the Doppler frequency is set as follows:

$$
f_{d}(t)=\left\{\begin{array}{cc}
-7500 t+f_{d 1} & 0 \leq t<3 \\
15000 t^{2}-7500 t+f_{d 2} & 3 \leq t<4 \\
7500 t+f_{d 3} & 4 \leq t<8
\end{array}\right.
$$

$f_{d 1}, f_{d 2}$ and $f_{d 3}$ represent the initial values of Doppler frequency at the beginning of three periods respectively. The received signal is a standard sinusoidal signal, which is expressed as follows:

$$
s(t)=A \cos \left(\left(f_{c}+f_{d}(t)\right) t+\Phi\right)+N(t)
$$

In formula (9), $A$ is signal amplitude, $f_{c}$ is carrier frequency, $\Phi$ is initial phase, $N(t)$ is additive Gauss white noise.

The simulation is divided into three stages according to the Doppler frequency change and parameter settings are shown in Table 1.

Table 1. Simulation parameter settings.

\begin{tabular}{|c|c|c|c|}
\hline Parameter & Value & Parameter & Value \\
\hline$f_{d 1}$ & $500 \mathrm{~Hz}$ & $f_{c}$ & $14 \mathrm{MHz}$ \\
\hline$f_{d 2}$ & $500 \mathrm{~Hz}$ & $f_{s}$ & $56 \mathrm{MHz}$ \\
\hline
\end{tabular}

\begin{tabular}{|c|c|c|c|}
\hline$\Phi$ & 0 & $\begin{array}{c}\text { Segment time } \\
\text { of Rife }\end{array}$ & $0.01 \mathrm{~s}$ \\
\hline$C / N_{0}$ & 50 & $\begin{array}{c}\text { Integral time } \\
\text { of loop }\end{array}$ & $0.001 \mathrm{~s}$ \\
\hline
\end{tabular}

\subsection{Simulation results}

\subsubsection{Stage one}

When PLL is used for tracking directly, $B_{L}=55 \mathrm{~Hz}$. The tracking results are shown in Figure 4.
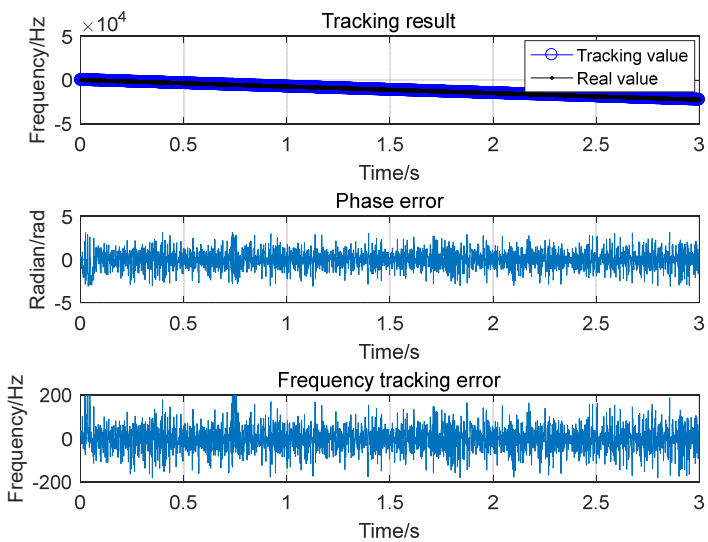

Figure.4. PLL direct tracking results

After fitting the tracking results, the Doppler frequency acceleration is $-7.486 \mathrm{KHz} / \mathrm{s}$, and the Doppler frequency initial value is $466.9 \mathrm{~Hz}$.

The fitting results of estimated frequency from Rife of 300 segment signals are shown in Figure 5.

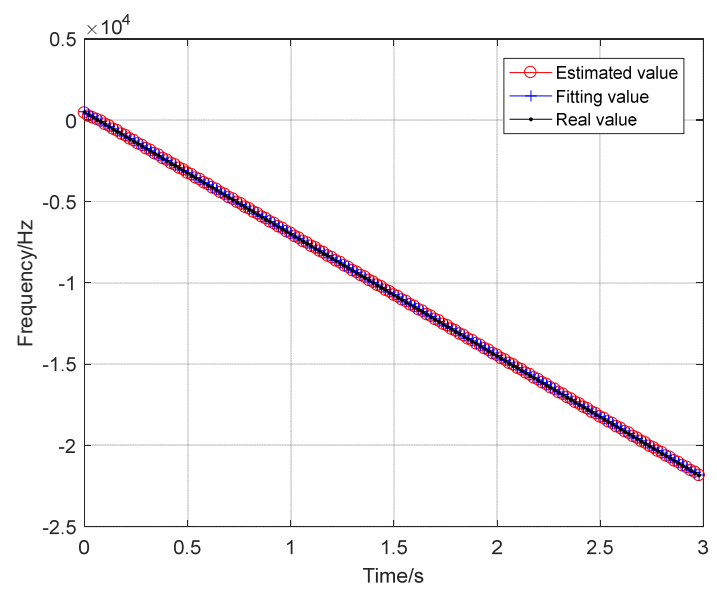

Figure.5. Frequency estimation results of Rife

The Doppler frequency acceleration estimation value is $-7.469 \mathrm{KHz} / \mathrm{s}$, and the Doppler frequency initial estimation value is $503.9 \mathrm{~Hz}$. After the signal is compensated, the PLL tracking results are shown in Figure 6. At this time, $B_{L}=5 \mathrm{~Hz}$.

After fitting the tracking results, the Doppler frequency acceleration is $-30.9 \mathrm{~Hz} / \mathrm{s}$, and the Doppler frequency initial value is $-3.88 \mathrm{~Hz}$. 

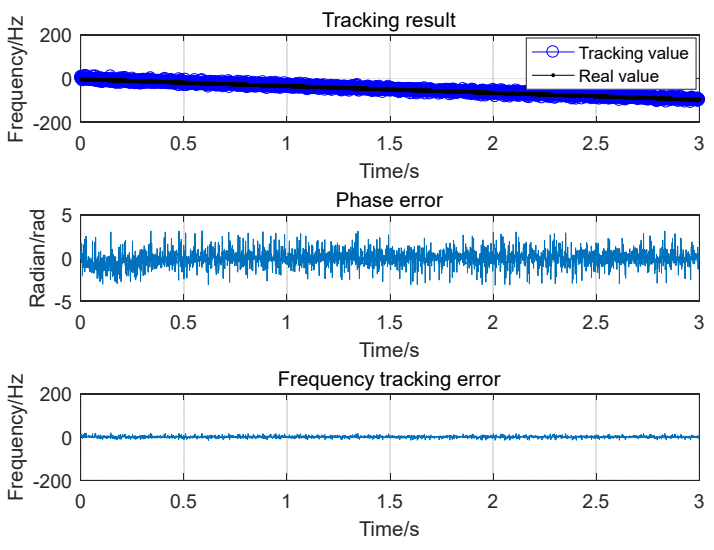

Figure.6. PLL tracking results after compensating

\subsubsection{Stage two}

When PLL is used for tracking directly, $B_{L}=60 \mathrm{~Hz}$. The tracking results are shown in Figure 7.
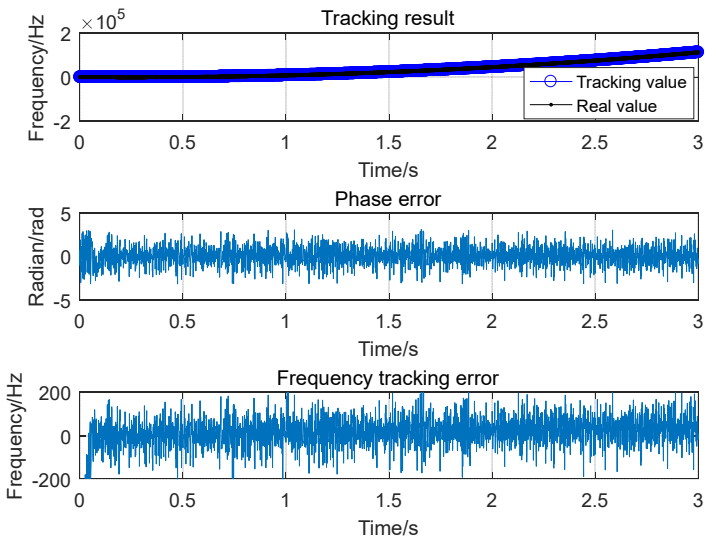

Figure.7. PLL direct tracking results

After second order polynomial fitting the tracking results, the Doppler frequency jerk is $14.987 \mathrm{KHz} / \mathrm{s}^{2}$, the Doppler frequency acceleration is $-7.437 \mathrm{KHz} / \mathrm{s}$, and the Doppler frequency initial value is $459.7 \mathrm{~Hz}$.

The fitting results of estimated frequency from Rife of 100 segment signals are shown in Figure 8.

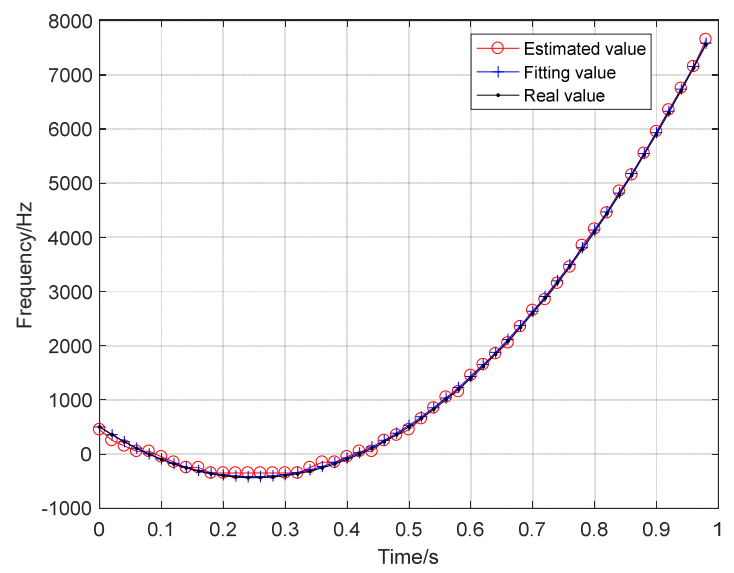

Figure.8. Frequency estimation results of Rife
The Doppler frequency jerk estimation value is 14 . $966 \mathrm{KHz} / \mathrm{s}^{2}$, acceleration estimation value is -7.407 $\mathrm{KHz} / \mathrm{s}$, and the initial estimation value is $499.7 \mathrm{~Hz}$. After the signal is compensated, the PLL tracking results are shown in Figure 9. At this time, $B_{L}=30 \mathrm{~Hz}$.
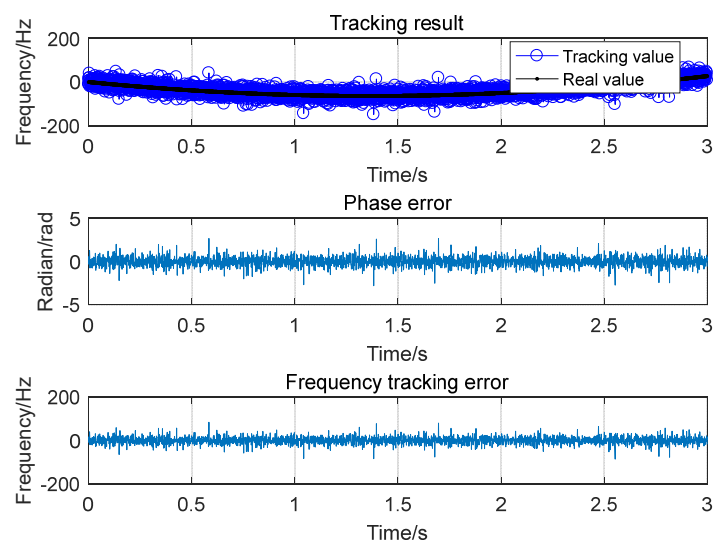

Figure.9. PLL tracking results after compensating

After fitting the tracking results, the Doppler frequency jerk is $33.91 \mathrm{~Hz} / \mathrm{s}^{2}$, acceleration is $-92.7 \mathrm{~Hz} / \mathrm{s}$, and initial value is $0.18 \mathrm{~Hz}$.

\subsubsection{Stage three}

The processing method of stage three is the same as stage one.

It can be seen from the above simulation results that the tracking accuracy is improved obviously and the steady phase error is smaller when there exists a jerk.

\subsection{Comparison of estimation accuracy of FLL and Rife for dynamic characteristic parameters}

Compared with the FLL-PLL frequency tracking method, the difference of the method proposed in this paper is that the open-loop frequency estimation (Rife algorithm) and the fitting method are used to eliminate most of the dynamic characteristics of the signal instead of FLL. Therefore, the final frequency tracking precision determined by FLL and Rife algorithms. The Doppler dynamic parameter estimation errors of FLL and Rife algorithms under different SNR are shown in Table 2 (1000 Monte Carlo simulations):

It can be seen from table 2 that when the SNR is low, the estimation accuracy of Rife algorithm is much higher than that of FLL. With the increase of SNR, the estimation accuracy of the two algorithms is improved significantly and tends to be stable. When the SNR is high, the dynamic parameter estimation performance of Rife algorithm has little difference with that of FLL.

Table 2. Doppler dynamic parameter estimation errors of FLL and Rife $\left(B_{L}=10 \mathrm{~Hz}\right)$

\begin{tabular}{|c|c|c|c|c|}
\hline$C / N_{0}$ & \multirow{2}{*}{ Algorithm } & $\begin{array}{c}\text { Initial } \\
\text { Value }(\mathrm{Hz})\end{array}$ & $\begin{array}{c}\text { Acceleration } \\
(\mathrm{Hz} / \mathrm{s})\end{array}$ & $\begin{array}{c}\text { Jerk } \\
\left(\mathrm{Hz} / \mathrm{s}^{2}\right)\end{array}$ \\
\hline
\end{tabular}




\begin{tabular}{|c|c|c|c|c|}
\hline \multirow{2}{*}{50} & FLL & 93 & 311 & 244 \\
\cline { 2 - 5 } & Rife & 5 & 83 & 65 \\
\hline \multirow{2}{*}{55} & FLL & 41 & 140 & 104 \\
\cline { 2 - 5 } & Rife & 4 & 83 & 61 \\
\hline \multirow{2}{*}{60} & FLL & 24 & 71 & 46 \\
\cline { 2 - 5 } & Rife & 4 & 83 & 61 \\
\hline \multirow{2}{*}{65} & FLL & 24 & 69 & 46 \\
\cline { 2 - 5 } & Rife & 4 & 83 & 61 \\
\hline \multirow{2}{*}{70} & FLL & 23 & 66 & 45 \\
\cline { 2 - 5 } & Rife & 4 & 83 & 61 \\
\hline
\end{tabular}

9. W.Q. Mou, R.K. Liu, Q.P. Song, et al. Journal of Beijing University of Aeronautics and Astronautics, 41(2015), 1918-1924.

10. H. Xun, X.R.. Tao, R. Tao, et al. Journal of Electronics \& Information Technology, 36(2014), 577-582.

11. J.R. Cheng, G.B. Liu, Q. Zhang, et al. Journal of Astronautics, 36(2015), 103-108.

12. Rife D C, Boorstyn R R. IEEE Trans Inform Theory, 20(1974), 591-598.

13. X.D. Wang, Y. Liu, Z.M. Liu. Systems Engineering and Electronics, 20(2008),621-624.

14. J.X. Liu. Spacecraft TT \& C and information transmission technology, National Defense Industry Press, 2011, 74-75.

15. L.D. Li.. Journal of Liaoning Technical University(Natural Science), 30 (2011),202-204.

16. C. Wang, H.P. Wang, W. Xiong, et al. Acta Aeronautica et Astronautica Sinica, 37(2016), 16031613.

\section{Conclusion}

This paper proposes an approved frequency tracking method in connected element interferometry. The estimation and compensation of Doppler dynamic parameters overcome the limitation of the dynamic stress on the traditional PLL tracking capability. Simulation results show that the accuracy of the new method is much higher than that of the traditional PLL method. At the same time, the dynamic parameter estimation performance of Rife algorithm is better than that of FLL under low SNR.

\section{References}

1. Edwards C. Goldstone intracomplex connected element interferometry. TDA Progress Report, $1990,42-101$.

2. L. Huang, H.T. Li, W.H. Hao. Journal of Spacecraft TT\&C Technology, 33(2014),371-376.

3. P. Lian. Optical Engineering,43(2004 ),1552-1560.

4. Kamel M, Borio D, Nielsen J, et al. The Institute of Navigation GNSS, San Diego, 2011, 374-383.

5. X. Zhang,C. Guo, J. Cao. International Conference on Communications, Circuits and Systems, 2014,243-246.

6. W. Li,S. Liu,C. Zhou,et al. High Dynamic Carrier Tracking Using Kalman Filter Aided Phase-Lock Loop,2007,673-676.

7. F. Shen, R. He, D.Z. Lv, et al. Journal of Astronautics, 33(2012), 1041-1047.

8. X.D. Deng, W. Sun. Modern Defence Technology, 38(2010), 137-141. 